Polymer Journal Vol. 9, No. 2, pp 227-230 (1977)

SHORT COMMUNICATION

\title{
Macrocyclic Formals. II. The Effect of Initiators and Solvents on the Two Stage Polymerization of 1,3,6,9-Tetraoxa-Cycloundecane
}

\author{
Yuhsuke KawaKami and Yuya Yamashita \\ Department of Synthetic Chemistry, Faculty of Engineering, Nagoya University, \\ Furo-cho, Chikusa-ku, Nagoya 464, Japan.
}

(Received December 21, 1976)

\begin{abstract}
KEY WORDS Cyclic Formals / Cationic Polymerization / Gel Chromatography / Cyclic Oligomer / Copolymerization / Styrene /
\end{abstract}

We have reported that 1,3,6,9-tetraoxacycloundecane is polymerized in two stages, forming cyclic oligomers at the first stage and high polymers at the second stage. ${ }^{1}$ In order to obtain a general idea about the nature of the active centers in the each stage, the effects of solvents, initiators, and the concentration of initiators on product distribution were studied. Copolymerization with styrene was also studied. It was found that the polymerization in nitromethane by boron trifluoride ether complex and polymerization by trifluoromethanesulfonic acid in dichloromethane proceeded almost instantaneously. However, the gel chromatograms of the reaction products are almost the same in all cases. This implies that even if the reaction conditions are changed, the principal reaction operating in the polymerization of 1,3,6,9-tetraoxacycloundecane is not altered.

\section{EXPERIMENTAL}

Solvents were purified and dried by the usual methods. The reactions were carried out under nitrogen atmosphere in a flask equipped with a three-way cock capped with a serum cap. The conversion of monomer was determined by gas chromatography on the sample pulled out with a syringe through the three way cock. The product distribution was determined by gel chromatography.

\section{RESULTS AND DISCUSSION}

The effect of changing solvents on the timeconversion relationship is shown in Figure 1. The rate of monomer consumption was affected very much by the nature of the solvents. The reaction in nitromethane is almost instantaneous,

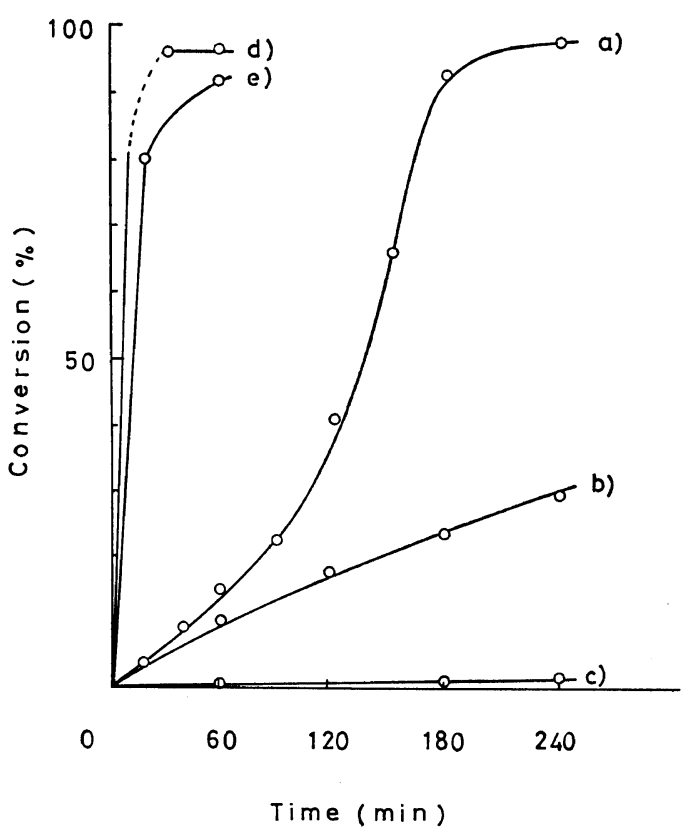

Figure 1. Time-conversion relationship in the polymerization of 11-CF-4 in various solvents: [11-CF-4], $3.25 \times 10^{-1} M$; $\left[\mathrm{BF}_{3} \cdot \mathrm{OEt}_{2}\right], 1.38 \times 10^{-2}$ $M ; 0^{\circ} \mathrm{C} ;$ a), dichloromethane; b), 1,2-dimethoxyethane; c), 1,3-dioxane; d), nitromethane; e), acetonitrile, 


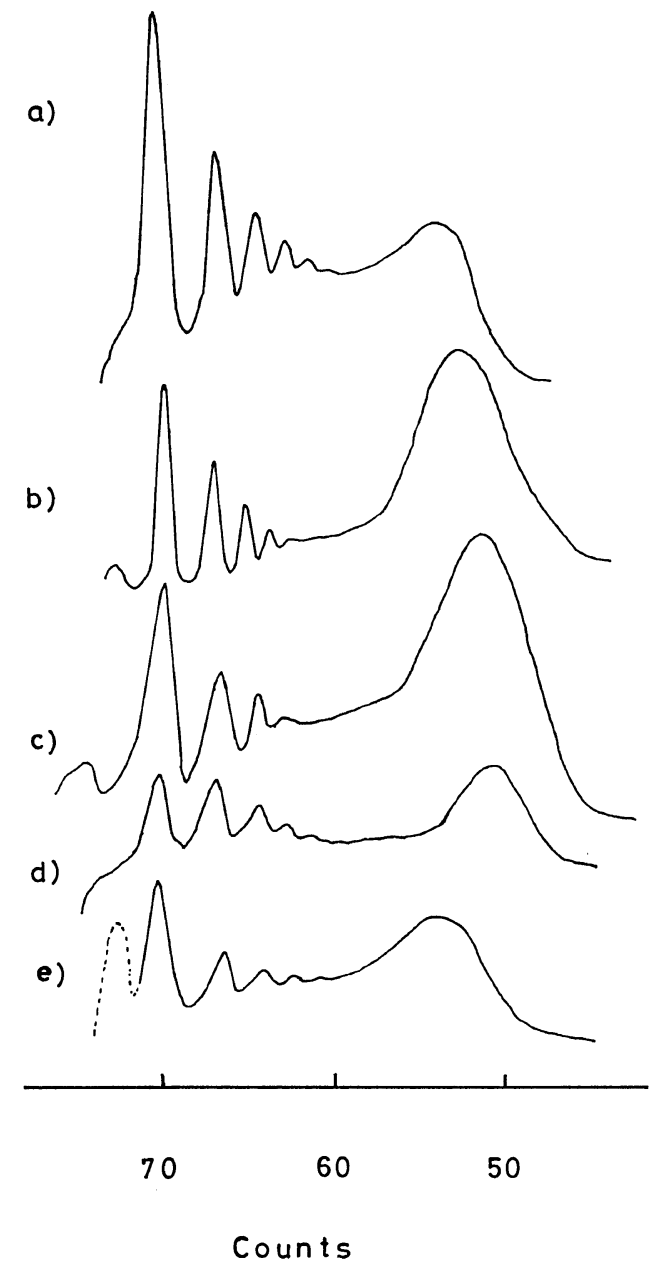

Figure 2. Gel chromatograms of reaction products in various solvents: [11-CF-4], $3.25 \times 10^{-1} M ;\left[\mathrm{BF}_{3}\right.$. $\left.\mathrm{OEt}_{2}\right], 1.38 \times 10^{-2} M ; 0^{\circ} \mathrm{C}$; a), dichloromethane; b), 1,2-dimethoxyethane; c), 1,3-dioxane; d), nitromethane; e), acetonitrile; reaction time, a) $24 \mathrm{hr}$, b) $40 \mathrm{hr}$, c) $70 \mathrm{hr}$, d) $24 \mathrm{hr}$, e) $24 \mathrm{hr}$.

but the product distribution is more or less the same as that in dichloromethane. This is shown in Figure 2. The reaction in acetonitrile is also fast. Contrary to this, the reactions in ether solvents are very slow. In dioxane, even after $27 \mathrm{hr}$ of reaction, the conversion is $21 \%$ and the only products are cyclic origomers.

The effects of intitiators on the time-conversion relationship are shown in Figure 3. The rate of monomer consumption is affected very much by initiators. Trifluoromethane-sul-

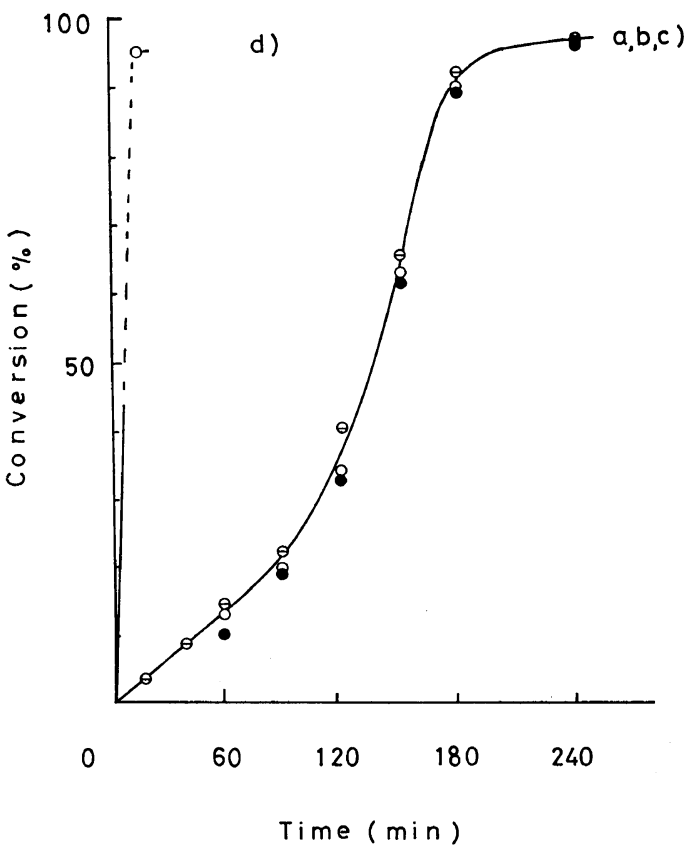

Figure 3. Time-conversion relationship in the polymerization of 11-CF-4 by various initiators in dichloromethane: [11-CF-4], $3.25 \times 10^{-1} M$; [initiator], $\left.\left.1.38 \times 10^{-2} M ; 0^{\circ} \mathrm{C} ; \mathrm{a}\right), \bigcirc \mathrm{BF}_{3} \cdot \mathrm{OEt}_{2} ; \mathrm{b}\right), \ominus$ $\left.\left.\mathrm{SnCl}_{4} ; \mathrm{c}\right), \mathrm{WCl}_{6} ; \mathrm{d}\right), \mathrm{CF}_{3} \mathrm{SO}_{3} \mathrm{H}$.

fonic acid initiator gives instantaneous polymerization. The rate of reaction by Lewis acids is quite similar to that by boron trifluoride ether complex. And the product distributions are again quite similar to each other. The fact that the changes in reaction conditions, such as the kinds of iniatiators and solvents, did not change the product distribution may indicate the presence of equilibrium concentrations for each cyclic oligomer.

Taking the fact that the reaction proceeds in two stages into account, it may be reasonable to consider that the accumulated active species which form cyclic oligomers in the first stage are responsible for producing the active species which form high polymers in the second stage.

In order to obtain a little more detailed information, the effect of the concentration of initiator was examined. The results are shown in Figures 4 and 5. Although the reaction proceeds faster with higher concentration of initiator, the conversion of monomer at the 


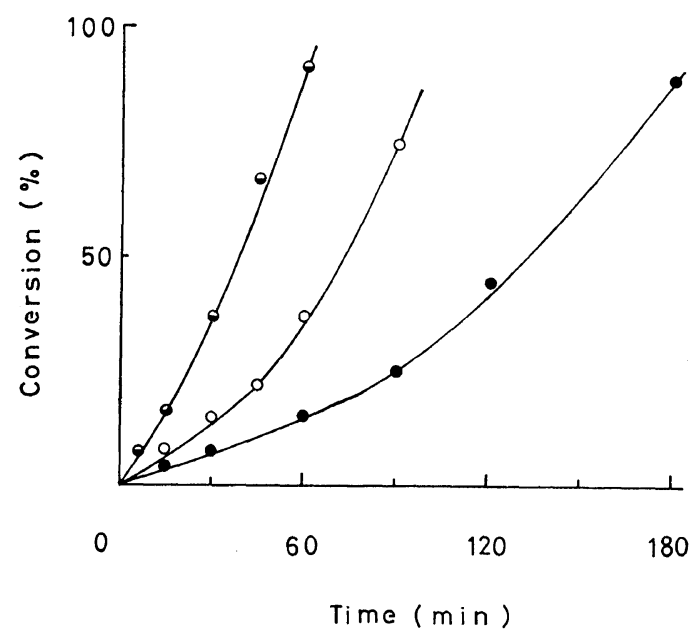

Figure 4. The effect of the concentration of initiator on the time-conversion relationship: [11CF-4], $3.25 \times 10^{-1} M$ in dichloromethane; $0^{\circ} \mathrm{C}$; $\left[\mathrm{BF}_{3}\right.$. $\mathrm{OEt}_{2}$ ], $1.38 \times 10^{-2} M, \bigcirc 2.76 \times 10^{-2} M, \bigcirc 4.14 \times$ $10^{-2} M$.

a)

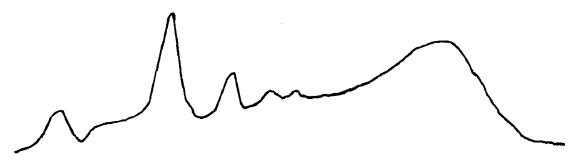

b)

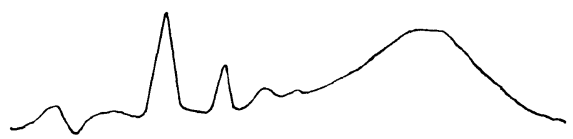

c)
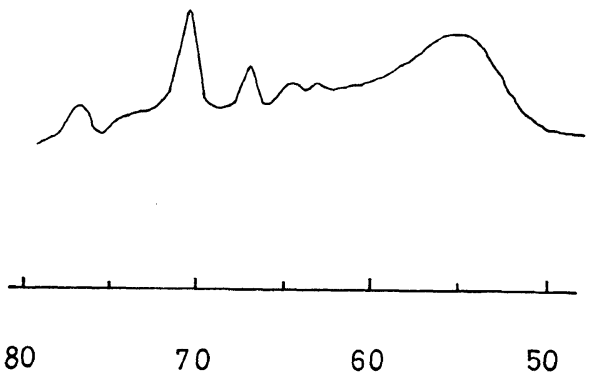

Counts

Figure 5. Gel chromatograms of reaction products with various concentrations of initiator, $0^{\circ} \mathrm{C}$ in dichloromethane: [11-CF-4], $3.25 \times 10^{-1} M$; $\left[\mathrm{BF}_{3}\right.$. $\left.\mathrm{OEt}_{2}\right]$, a) $1.38 \times 10^{-2} M$, b) $2.76 \times 10^{-2} M$, c) $4.14 \times$ $10^{-2} M$. inflection point from the first stage to second stage does not change when the same original concentration of monomer is used. Furthermore, the product distribution is almost the same even with the change of the concentration of initiator. This fact also suggests the presence of the equiilibrium concentrations of cyclic oligomers.

It is reported that 1,3-dioxolane is copolymerized with styrene. ${ }^{2}$ This is attributed to the carbocationic nature of the propagating species of 1,3-dioxolane polymerization.

In order to obtain a general idea about the reactive species in 1,3,6,9-tetraoxacycloundecane polymerization by boron trifluoride ether complex, the copolymerization with styrene was studied. The time-conversion relationship and the change in gel chromatogram in the copolymerization with styrene are shown in Figures 6 and 7 . We can clearly see that styrene reacts only in the second stage, copolymerizing with 1,3,6,9-tetraoxacycloundecane, and not in the first stage. There were not any co-oligomers

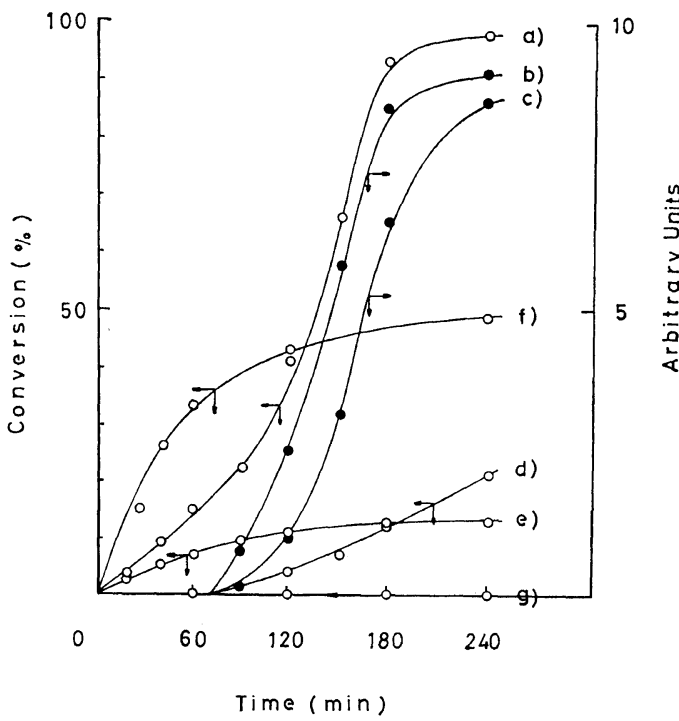

Figure 6. Time-conversion relationship in the copolymerization of $11-\mathrm{CF}-4$ with styrene $0^{\circ} \mathrm{C}$ in dichloromethane: $\left[\mathrm{BF}_{2} \mathrm{OEt}_{2}\right], 1.16 \times 10^{-2} \mathrm{M}$; [11CF-4], 3.81 $\times 10^{-1} M$; [St], 3.70 $\times 10^{-1} M$; a), 11-CF-4; b), the peak area of polymer monitored by RI; c), the peak area of polymer monitored by UV absorption at $254 \mathrm{~nm}$; d), styrene; e), cyclic dimer of 11-CF-4; f), styrene in homopolymerization; g), styrene in oligomers. 
1) a)

b)

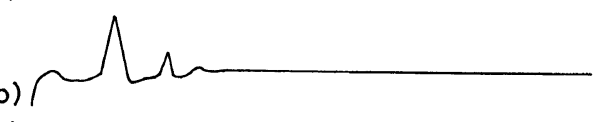

2)

3)

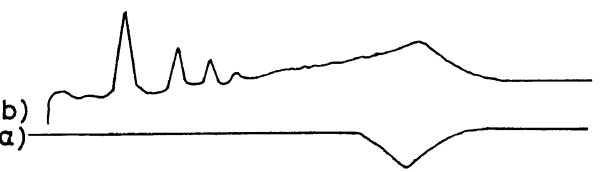

b)

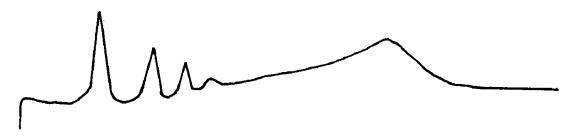

4)

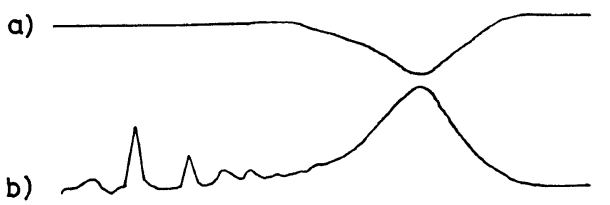

5)

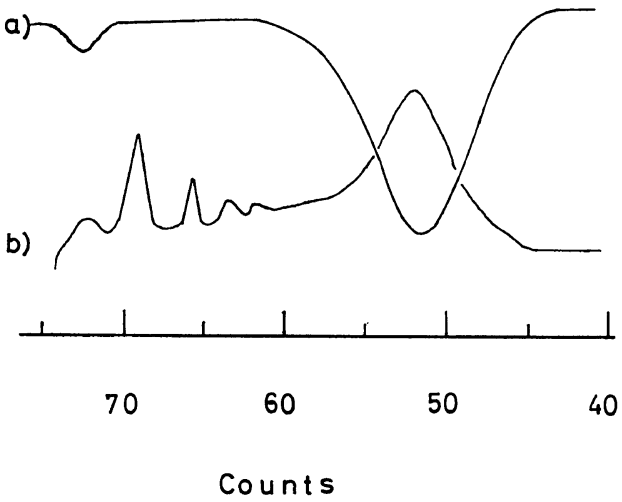

Figure 7. Change in gel chromatograms in the course of reaction time in the copolymerization of 11-CF-4 with styrene $0^{\circ} \mathrm{C}$ in dichloromethane: [11-CF-4], $3.81 \times 10^{-1} M$; [St], $3.70 \times 10^{-1} M ;\left[\mathrm{BF}_{3}\right.$. $\mathrm{OEt}_{2}$ ], $1.16 \times 10^{-2} M$; a), monitored by UV; b), monitored by RI; reaction time; 1) $40 \mathrm{~min}$, 2) 90 min, 3) $120 \mathrm{~min}$, 4) $150 \mathrm{~min}$, 5) $180 \mathrm{~min}$. detected in the first stage. The polymer formed in the copolymerization with styrene was proved to be a random copolymer by fractional reprecipitation from benzene into methanol and from benzene into ethyl ether, checked by gel chromatography and NMR.

These facts may be best understood as follows:

1) Initiator and monomer form the active species in the first stage, which is presumably a tertiary oxonium ion. This active species is responsible for the formation of cyclic oligomers.

2) The active species in the second stage are formed by reactions which are unknown, at present, from the species accumulated in the first stage. And they have a carbocationic nature and are responsible for the high polymerization of 1,3,6,9-tetraoxacycloundecane and the copolymerization with styrene.

\section{REFERENCES}

1. Y. Kawakami, Y. Yamashita, and K. Kitano, J. Polym. Sci., Polym. Lett. Ed., in press.

2. a) M. Okada, Y. Yamashita, and Y. Ishii, Makromol. Chem., 80, 196 (1964).

b) Y. Yamashita, M. Okada, and M. Hirota, ibid., 122, 284 (1969). 